\title{
THE 1964 IAU SYSTEM AND THE GEODETIC
}

\section{REFERENCE SYSTEM 1967}

\author{
J. KOVALEVSKY \\ Bureau des Longitudes, Paris, France
}

\begin{abstract}
(Presented at IAU Colloquium No. 9, 'The IAU System of Astronomical Constants', Heidelberg, Germany, August 12-14, 1970.)
\end{abstract}

In 1964, the IAU adopted, among the primary constants of its system, the following three constants especially related to the shape, dimensions and gravitational field of the Earth:

$$
\begin{aligned}
& a \quad=6378160 \mathrm{~m} \\
& G M=398603 \times 10^{9} \mathrm{~m}^{3} / \mathrm{s}^{2} \\
& J_{2}=0.0010827
\end{aligned}
$$

These values were discussed in 1967 by the IAG and adopted by the IUGG as the basis of a 'Geodetic Reference System 1967'. Although at that time better values of these constants were available, IUGG decided to adopt the values of the IAU system in order to keep consistency with the values agreed upon by the astronomers.

This fact gives to the IAU system a value that has a wider circulation in the scientific community than just astronomers. This gives to the IAU a certain inter-union responsibility in the field.

IUGG instructed IAG to prepare values of all relevant parameters representing the standard ellipsoid with these 3 basic characteristic parameters. These values have to be consistent to the 12 th significant figure in such a way that geodetic computations on the reference ellipsoid should be good to one tenth of a millimeter. The results of the work carried out independently by several groups are being published in a special issue of 'Bulletin Géodésique' to appear soon, called 'Geodetic Reference System 1967 '.

In order to compute these derived parameters, a mean rotational speed of the Earth at $1900.0, \omega$, had to be defined. It is:

$$
\omega=\frac{2 \pi}{86400} \times \frac{s+86400-\mu / 1500}{s}=7.2921151467 \times 10^{-5} \mathrm{rad} / \mathrm{s}
$$

where $s$ is the number of ephemeris seconds in a tropical year at 1900.0, and $\mu=p \cos \varepsilon-12.473 \sin ^{2} \varepsilon, p$ being the constant of precession, and $\varepsilon$ the obliquity of the ecliptic.

Assuming that the atmosphere is concentrated on the surface of the ellipsoid, one gets the following results: 


$$
\begin{array}{ll}
\text { Reciprocal flattening } & 1 / f=298.247167427 \\
\text { semi-minor axis } & b=6356774.5161 \mathrm{~m} \\
\text { normal gravity at equator } & \gamma_{\mathrm{e}}=978.03184558 \mathrm{gal} . \\
\text { gravity formula } & \gamma=\gamma_{\mathrm{e}} \frac{1+k \sin ^{2} \varphi}{\sqrt{1-e^{2} \sin ^{2} \varphi}}
\end{array}
$$

with $k=0.00193166338321$

and $e^{2}=0.00669460532856$

These numbers are pure computational consequences of the IAU system. They are different from those published in the IAU list of secondary constants, which were rounded. The International Association of Geodesy requests that its more precise values should be used in preference to any others. I concur with this request, provided it is clearly stated that these numbers represent an Earth Standard Model, and suggest that the IAU endorses the IUGG values, just as the IUGG endorsed the IAU system of fundamental constants. 